\title{
QOS MULTICAST ROUTING BASED ON A HEURISTIC GENETIC ALGORITHM
}

\author{
Mehdi Karabi \\ Computer Engineering \\ Department, Iran University of \\ Science and Technology, Tehran, \\ Iran \\ mehdikarabi@iust.ac.irT
}

\author{
Mahmood Fathy \\ Computer Engineering \\ Department, Iran University of \\ Science and Technology, Tehran, \\ Iran \\ mahfathy@iust.ac.ir
}

\author{
Mehdi Dehghan \\ Computer Engineering \\ Department, Amirkabir University \\ of Technology, Tehran, Iran \\ dehghan@ce.aut.ac.ir
}

\begin{abstract}
The problem of computing the Bandwidth-DelayConstrained least-cost QOS multicast routing is an NPComplete problem. We present a new efficient heuristic algorithm for the QOS multicast routing that depends on bounded end-to-end delay and link bandwidth along the path from the source to each destinations, and minimum cost of Steiner tree. In this algorithm, the modified Determinant Encoding is used for genotype representation. The major objectives of this study are: To modify the determinant encoding to be suitable for the QOS-constrained Steiner tree problems; To propose a new algorithm for repairing determinant codes for Steiner tree algorithms; To develop a new heuristic GAbased solution to the QOS multicast routing problem with modified determinant encoding and new proposed repair function. To compare the performance of the proposed algorithm with some existing GA-based algorithms, we simulate our algorithm for several networks with different network and multicast sizes. These results show that our proposed algorithm can generate a smaller average tree cost than the mentioned existing algorithms within a reasonable time.
\end{abstract}

Keywords: Multicast routing; Genetic Algorithm; Quality of Service.

\section{INTRODUCTION}

Multicasting involves the transport of the same information from a sender to multiple receivers along a multicast tree. In the past much work has been focused on algorithms for computing low-cost multicast trees. Since the problem of the computing minimum-cost multicast trees (called Steiner trees) in a network is NPcomplete [7]. Most of the proposed algorithms are heuristic. Some of well-known Steiner tree heuristics are KBM [1] and BSMA [1]. methods based on neural networks and genetic algorithms [2] have also been proposed for computing low-cost multicast trees. As more and more bandwidth-delay-sensitive networking applications such as video conferencing, tele-education and many other distributed multimedia applications appear, efficient of low-cost multicast tree cause more satisfaction of users. Bandwidth-delay-constraint is of great interest. In the last few years, a lot of bandwidthdelay-constrained multicast routing heuristics have been proposed [1, 2]. The simulation results given in [8] have shown, however, that most of the proposed algorithms either work too slowly or can not compute bandwidthdelay-constrained multicast trees with low-cost.

Deterministic computation of low-cost bandwidthdelay-constrained multicast trees usually requires large amount of computation time. For this reason, methods based on neural networks or genetic algorithms may be more helpful. In this paper, we propose a novel QoSbased multicast routing algorithm based on GA. The modified determinant encoding is used for genotype representation. We evaluate the performance of the proposed algorithm by result of simulation.

The remainder of this paper is organized as follows: The problem description and formulation is given in section 2. In Section 3, we describe the proposed algorithms. We then evaluate the convergence of the proposed GA-based algorithms. Section 4 gives the performance evaluation of the proposed algorithms and the comparison of them with other similar algorithms.

\section{PROBLEM DESCRIPTION AND FORMULATION}

A network can be modeled as a graph $G(V, E)$, where $V$ is the set of all nodes, representing routers or switches; $E$ is the set of edges, representing physical or logical connectivity between nodes. Each link is bidirectional, i.e. the existence of a link $e=(u, v)$ from node $u$ to node $v$ 
implies the existence of another link $e^{\prime}=(v, u)$ for any $u$, $v \in V$. Any link $e \in E$ has a cost $\operatorname{cost}(e): E \rightarrow R_{+}$, a delay $\operatorname{delay}(e): E \rightarrow R_{+}$, and a bandwidth bandwidth(e): $E \rightarrow R_{+}$ associated with it, where $R_{+}$is the set of positive real numbers. The function cost(.) defines the measure to be minimized. The functions delay(.) and bandwidth(.) define the measure to be bounded. Let $s \in V$ be the multicast source, $M \subseteq V$ - $\{s\}$ multicast destinations. A multicast tree $T(s, M)$ is a tree rooted at $s$ and spanning all members of $M$. The total path delay from $s$ to any destination $d$, denoted by $\operatorname{delay}\left(P_{t}(s, d)\right)$, is simply the sum of the delay of link along $P_{t}(s, t)$, i.e.

$$
\operatorname{delay}(\operatorname{Pt}(s, d))=\sum_{e \in P_{t}(s, d)} \operatorname{delay}(e)
$$

The total cost of the tree, denoted by $\operatorname{Cost}(T)$, is defined as sum of the cost of links in the tree, i.e.

$$
\cos t(T)=\sum_{e \in T} \cos t(e)
$$

The bandwidth from $s$ to any destination $d$, denoted by $\operatorname{bandwidth}_{t}\left(P_{t}(s, d)\right)$, is defined as the minimum of the bandwidth among links along $P_{t}(s, d)$, i.e.

$$
\text { Bandwidth }\left(P_{t}(s, d)\right)=\min \left\{\text { bandwidth }(e), e \in P_{t}(s, d)\right\}
$$

Let $\Delta_{d}$ be the delay constraint and $B_{d}$ the bandwidth constraint of the destination node $d$. The bandwidthdelay-constrained least-cost multicast routing problem is defined as minimization of $\operatorname{Cost}(T(s, M))$ subject to:

$$
\left\{\begin{array}{l}
D\left(P_{T}(s, d)\right) \leq \Delta_{d}, \forall d \in M \\
B\left(P_{T}(s, d)\right) \geq B d, \forall d \in M
\end{array}\right.
$$

\section{THE PROPOSED MULTICAST ROUTING ALGORITHM}

The genetic algorithms have emerged as a powerful tool for solving NP-complete problems in many research areas [5]. A simple genetic algorithm works according to the following steps:

S1: Select encoding scheme and generate initial population.

S2: Evaluate the fitness of every individual.

S3: Produce a new generation of population based on the fitness of individual (by using genetic operators like crossover and mutation).

S4: Evaluate the fitness of every individual in the new generation.

S5: Continue steps S3 and S4 until a given criterion is satisfied.

\subsection{Genotype}

We modify the determinant encoding used in [4] for minimum spanning tree algorithm, such that it can be used in the GA-based algorithm to solve the QoS- constrained multicast tree problems. We define the degree matrix of graph $G$, denoted by Degree $(G(V, E))$, as follows:

$$
D(i, j)=\left\{\begin{array}{cc}
d(i) & i=j \\
-k & i \neq j
\end{array}\right.
$$

where $d(i)$ is the degree of node $i$, and $k$ is the number of edges from node $i$ to node $j$ in $G$. We define the Edgerange vector $R$ of a digraph $G$ as follows:

$$
R(j)=\{i \mid D(i, j)=-1\} \text { for } j=1 \text { to } n \text {. }
$$

where $D$ is the degree matrix and $n$ is the number of nodes in $G$. furthermore, we define Modified Determinant Encoding (MDC) as a string of $n-1$ integers in $\{0,1,2, . ., n\}$ that the $(j-1)^{t h}$ position in the string is randomly selected from $R(j)$, $($ for $j=2$ to $n)$. If the $(j-1)^{\text {th }}$ of MDC is $w$ it means the existence of an edge from node $w$ to node $j$ in $G$.

Fig. 1 shows a degree matrix, edges-range vector and obtained MDC of the Steiner tree for a given network graph. (a)

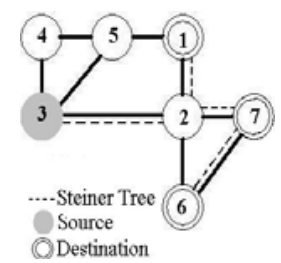

(b)

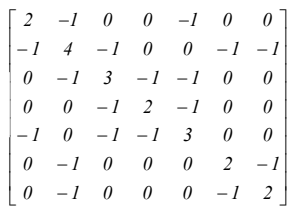

(c)

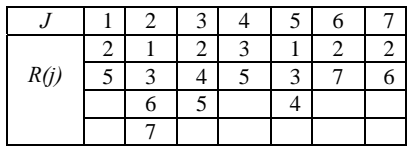

(d)

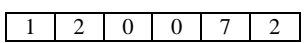

Fig. 1: (a) the digraph $G(V, E)$, (b)Degree matrix of $G$, (c) Edge-range vector of digraph $G(V, E)$, and (d)modified determinant encoding corresponding to the Steiner tree in $G$.

MDC scheme some times produces graphs, instead of Steiner trees. Our algorithm for repairing determinant codes is described as follows:

Repair Function: MDC may be illegal due to 3 reasons: 1) missing node “1", 2) self loops or cycles, 3) partitioning to some sub-trees. The algorithm proposed below describes a strategy to solve all of the three situations that lead to illegal Steiner trees.

$S 1$ - Repair missing node "1": if node $l \in M \cup\{s\}$ is not already in the code, replace a randomly selected allele with node "1", otherwise goto $S 2$.

S2- Repair self loops: for a given encoded string C, find $x$, such that $x=C(x)$. If any $x$ exists, set $C(x)=0$, otherwise goto $S 3$.

$S 3-$ Exam and repair cycles: repair operator initially tries to find existing cycles by using a grouping algorithm, and then repairs them. The Exam and Repair algorithm is shown in Fig. 2. 
S4- Connect sub-trees: repeat the following steps until a multicast group is formed:

a) Select randomly two sub trees from the sub trees formed in MDC

b) Find an edge $(i, j)$ such that node $i$ is in $g_{I}$ and node $j$ is in $g_{2}$. if such an edge exists set $C(j-1)=i$, otherwise goto step a.

c) Rename sub trees $g_{1}$ and $g_{2}$ in to $g_{l}$

S5-Finally remove all the leaves that are not member of Steiner tree.

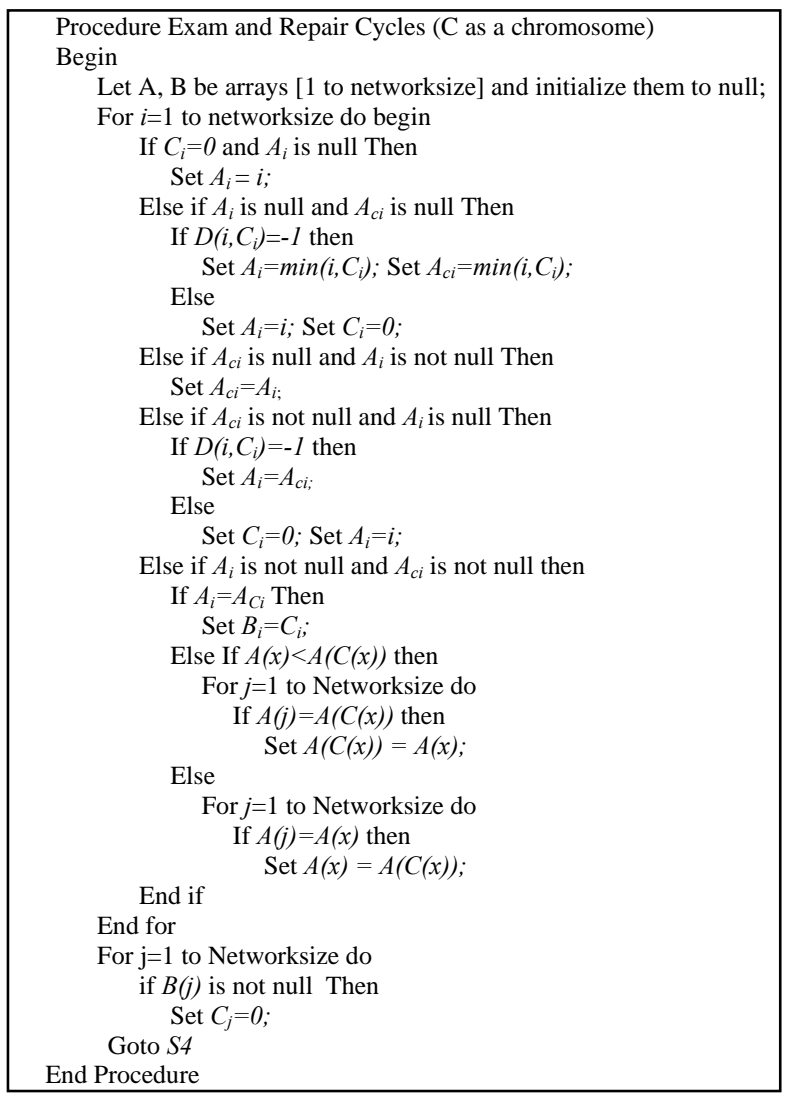

Fig. 2. Proposed Examining cycles and repairing algorithm

The construction of the chromosome corresponding to the multicast tree of Fig. 1 is described in table 1.

Table 1. Stages involved in construction MDC for the Steiner tree in Fig. 1.

\begin{tabular}{|l|r|r|r|r|r|r|}
\hline \multicolumn{1}{|c|}{ Description } & \multicolumn{5}{|c|}{ MDC } \\
\cline { 2 - 7 } & 1 & 2 & 3 & 4 & 5 & 6 \\
\hline $\begin{array}{l}\text { Initial MDC: } j-1 \text { position in MDC selected } \\
\text { from } R(j)\end{array}$ & 6 & 5 & 3 & 3 & 7 & 2 \\
\hline Repair missing node 1 $(S 1)$ & 1 & 5 & 3 & 3 & 7 & 2 \\
\hline $\begin{array}{l}\text { Checking cycles: vectors A is 1133322 and B is } \\
----3--\end{array}$ & 1 & 5 & 3 & 3 & 7 & 2 \\
\hline Repair cycles: $B(5)$ is not null, $C_{3}=0 .(S 3)$ & 1 & 0 & 3 & 3 & 7 & 2 \\
\hline Connect obtained sub trees in MDC. $(S 4)$ & 1 & 2 & 3 & 3 & 7 & 2 \\
\hline Remove extra leaves. (S5) & 1 & 2 & 0 & 0 & 7 & 2 \\
\hline
\end{tabular}

\subsection{Initial Population}

3.2.1 Pre-processing phase. Before starting the GA we can remove all links which their bandwidths are less than minimum required bandwidth constraint. If in the refined graph the source and all destination nodes are not in a connected sub-graph, this topology does not meet the bandwidth constraint, and otherwise we will use this subgraph as the network topology in our algorithm.

3.2.2 Initial population. Random initialization is used in this study. Each Allele in position $j-1$ of MDC, is randomly selected from $R(j)$, and then the repair procedure described in section 3.2.1 is performed on illegal chromosomes.

\subsection{Fitness Function}

The fitness function in our study is a scheme proposed in [1]. The fitness function uses the penalty technique, as follows:

$$
\begin{gathered}
F(T(s, M))=\frac{\alpha}{\sum_{e \in T(s, M)} C(e)} \prod_{d \in M} \phi\left(D(P(s, d))-\Delta_{d}\right) \prod_{d \in M} \phi\left(B(P(s, d))-B_{d}\right) \\
\phi(z)= \begin{cases}1 & z \leq 0 \\
\gamma & z>0\end{cases}
\end{gathered}
$$

where $\alpha$ is a positive real coefficient, $\phi(z)$ is the penalty function and $\gamma$ is the degree of penalty ( $\gamma$ is considered equal to 0.5 in our study).

\subsection{Selection}

The selection process used here is elitism model based on spanning the roulette wheel popsize times. The probability $P_{i}$ that a parent $T_{i}$ is selected, is given by

$$
p_{i}=\frac{F\left(T_{i}\right)}{\sum_{j=1}^{p o p-s i z e} F\left(T_{j}\right)}
$$

where $F\left(T_{i}\right)$ is the fitness of the individual $T_{i}$.

\subsection{Crossover and Mutation}

We have compared standard crossovers such as onepoint, two-points, and uniform crossover, and Insert and exchange mutations. In this study the uniform crossover and insert mutation used for crossover and mutation operations.

\subsection{Analysis of Convergence}

According to the Theorem 2.7 in Ref. [2], the GAbased algorithms proposed in this paper could finally converge to the global optimal solution. For a large-scale network, it is time-consuming to obtain the optimal 
solution to the bandwidth-delay-constrained least-cost multicast routing problem, which is NP-complete. This problem can be overcome by setting an appropriate iteration time of the genetic algorithm. In this way, we can obtain a near-optimal solution within a reasonable time limit.

\section{EXPERIMENTAL RESULTS}

In this section, we have used the simulation experiments to compare the performance of the proposed GA-based algorithms with the heuristic BSMA heuristic algorithm and some existing GA-based algorithms. We have implemented more than 2,000 lines Pascal program to simulate the proposed algorithm. All simulation experiments are run on a Pentium II 333, 256 MB RAM, IBM PC. The multicast group is randomly selected in the graph. The size of multicast group is considered $5 \%$ and $30 \%$ of the number of network nodes.

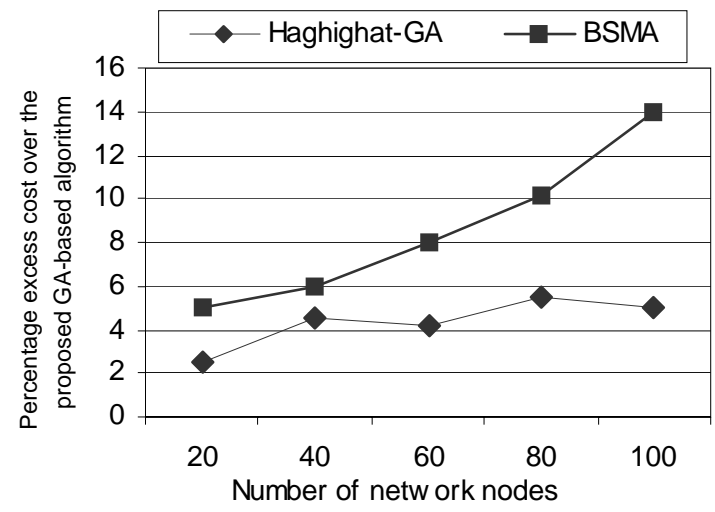

Fig. 3. Percentage excess cost over the proposed GA-based algorithm versus number of network node (multicast group size is $5 \%$ of number of network nodes).

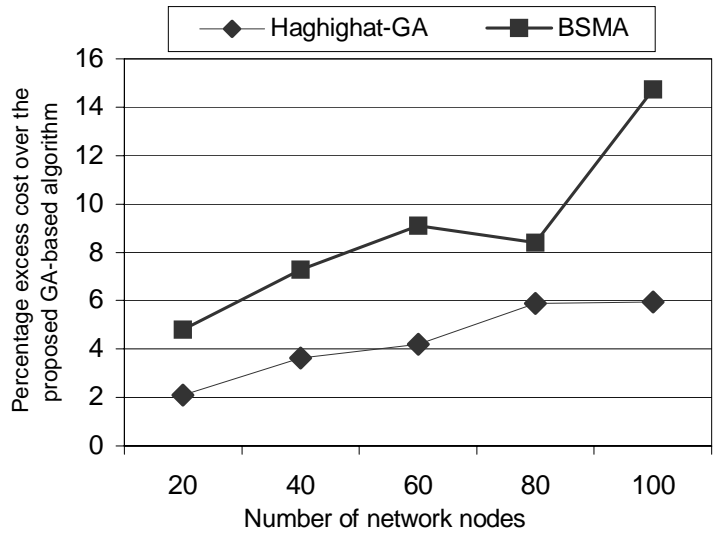

Fig. 4. Percentage excess cost over the proposed GA-based algorithm versus number of network node (multicast group size is $30 \%$ of number of network nodes).
We have tuned the proposed GA-based algorithms and the following parameters setting are achieved: crossover probability $P_{c}=0.95$ for uniform crossover, mutation probability $P_{m}=0.01$ for insert mutation.

Fig. 3 and Fig. 4 show the percentage tree cost of BSMA [7], Haghighat GA-based algorithm [1] comparison with our proposed GA-based heuristic algorithm for different network sizes and different multicast group sizes. These Figures show that our proposed GA-based heuristic algorithm can result in a smaller average tree cost than the mentioned existing algorithms.

\section{Acknowledgements}

This work was supported by Iran Telecommunication Research Center.

\section{References}

[1] A. T. Haghighat, K. Faez, M. Dehghan, A. Mowlaei, and Y. Ghahremani, "Efficient Multicast Routing with Multiple QoS Constraints Based on Genetic algorithms," IEEE SoftCom 2002, Crovatia, 8-11 Oct. 2002.

[2] J. J. Wu and R. H. Hwang, H. I. Lu, "Multicast routing with multiple QoS constraints in ATM networks," Information Sciences, Vol. 124, pp. 29-57, 2000.

[3] C. C. Palmer, "An approach to a problem in network design using genetic algorithms, " Ph.D. Dissertation in Computer Science, Polytechnic University, April 1994.

[4] N. Abuali, L. Wainwright, and A. Schoenefeld, " Determinant Factorization: A New Encoding Scheme for Spanning Tree Applied to the Probabilistic Minimum Spanning Tree Problem," Proceedings of the sixth International Conference on Genetic Algorithm (ICGA), Pittsburgh, PA, pp. 470-477, 1995.

[5] M. Gen and R. Cheng, Genetic algorithms and engineering optimization, John Wiley \& Sons, 2000.

[6] M. Gray and D. Johnson, " Computers and Intractability: A Guide to Theory of NP-Completeness, " New York: W. H. Freeman and Co., 1979.

[7] M. Parsa, Q. Zhu, and j.j. Garcia-Luna-Aceves, "An Iterative Algorithm for Delay-Constrained Minimum-Cost Multicasting, " IEEE/ACM Transactions on Networking, Vol. 6, No. 4, pp. 461-474, 1998.

[8] H. F. Salama, D. S. Reeves, and Y. Viniotis, "Evolution of Multicast Routing Algorithms for Real-Time Communication on High-Speed Networks," IEEE JSAC, Vol. 15, No. 3, Apr. 1997, pp. 332-345. 